ber 30 th it died, and an examination of the body was made on the same day, when the following unexpected and unusual circumstances presented themselres.

$\Lambda$ normal and well-developed uterus, wilh its Fallopian tubes and ovaries, was present. The canal leading from the external opening was the vagina, about two lines in diameter, perfectly cylindrical and patent. There was no urethra, but the neck of the bladder was inserted into the vagina. That this canal was the ragina, although not perfectly normal, is proved from these circumstances, First, the direct course of the canal was to the uterus, the bladder being connected with it at an obtuse angle. Secondly, the structure of its walls was that of the vagina, consisting of a mucous membrane, a dense layer of coloured fibrous tissue, and some condensed cellular membrane. This structure was continued beyond the insertion of the bladder into the vagina, even to the neck of the uterus. The anormal condition of this ragina was its not terminating by a cylindrical opening, around which a fibrous layer might be located. The above-mentioned structure, also, did not proceed to the termination of the canal, but only its most internal part, about twelve lines. The more external eight lines were composed of mucous membrane and cellular structure only ; but although the lower part of this canal bore some resemblance to the urethra, yet the circumstances above named will probably prove it to have been the vagina,

Neither testes nor spermatic cord could be discovered. The labia consisted of a portion of integument and cellular tissue folded upon itself, the two layers being very rearlily separated from each other.

This case appears to be interesting for three reasons:- -

First. From the great similarity of the ex. ternal parts to the male organs of generation, which was infuitely greater than is usually observed in anormal conditions of the female organs.

Secondly. From the canal consisting of vaginal tissue, with an urethral termination, the external opening also being anormal ; aud more especially from the direct insertion of the bladder into the vagina, causing the absence of the urethra.

Thirdly. It is illustrative of the remark made by Geoffroy St. Hilaire, that the organs of both sexes are originally formed on the same plan. It also shows how purely speculative must be the opinion of medical men in such anormal cases.

Birmingham, Nov, 1, 1813.

Fissures of the Anus.-For these MIMI. Bretonneau and Tronsseau recommend enemata of four sciuples of extract of rhatany to half a pint of water to be thrown up the rectum once or twice daily. In a few days the pain ceases, and the fissure heals.
DOUBLE BRAIN.

To the Editor.-Sir: On October 17th my attendance was requested on Mary Nelson, atat, 32, who on that day was seized with labour pains of hel seventh child. On arriv. ing at the house, and making an examina. tion, I found the os uteri fully dilated, and the pains strong and constant. The verlex presented, with the face towards the left ilium. Upon the head descending into the cavity of the pelvis I becare aware of the existence of a tumour, the nature of which I could not at first distinguish. The pains, however, soon expelled the child, when I found the tumour to be attached to the occi. pital bone, and of the size of a lurge orange, of a soft nature, and totally devoid of hair, although hair was plentiful upon the head. In conuection with this the child had spina bifida of the third, fourth, and fifth dorsal vertebra, and the right foot was clubbed. The other organs were normal. The child was seized with convulsions immediately after its birth. These continued, at intervals, until its death, which took place on the fol. lowing evening. Permission being given, I opened the tumour on the morning after. wards, and found it to contain a perfectly. developed brain, surrounded by its ordinary membranes, and connected with the poste. rior part of the cerebrum within the skul, through a circular opening of about an inch in diameter, just above the occipital pro. tuberance, and having, also, a distinct connection with the spinal cord, the nerves being given off in the usual manner. I am, Sir, yours respectfully,

Northwich, Nov. 1, 1843.

E. J. Barker.

THE STRUCTURE OF TENDON.

\section{THE QUESTION OF THE UNION} OF TENDON AND MUSCLE.

\section{To the Edilor of The Lancet.}

SrR,--The question has long been agitated in what manner the union between tendon and muscle is effected, and notwithstanding the valuable researches that have been made of late years, we do not yet seem to have come to a satisfactory conclusion on the sub. ject. This appears, in great measure, to have arisen from the fact that the inquiry has been directed rather to the immediate union of the two structures than to that of their elementary parts.

Each primitive fasciculus of muscular fibrillæ has been found by Mr. Bowman to be enclosed in a delicate sheath, which lie has named the sarcolemma, separate and dis. tinct from that which surrounds the larger fasciculi and the entire muscle. Now, the cellular tissue from which the external sheath is derived is represented as being continuous throughout the entire body; and from the 